\title{
Entropy
}

ISSN 1099-4300

(C) 2003 by MDPI

www.mdpi.org/entropy

\section{On the Measure Entropy of Additive Cellular Automata $f_{\infty}$}

\section{Hasan Akın}

Arts and Sciences Faculty, Department of Mathematics, Harran University; 63100, Şanlıurfa, Turkey. E-mail: akinhasan@harran.edu.tr

Received: 5 February 2003 / Accepted: 12 June 2003 / Published: 30 June 2003

\begin{abstract}
We show that for an additive one-dimensional cellular automata $f_{\infty}$ on space of all doubly infinitive sequences with values in a finite set $\mathrm{S}=\{0,1,2, \ldots, \mathrm{r}-1\}$, determined by an additive automaton rule $f\left(\mathrm{x}_{\mathrm{n}-\mathrm{k}}, \ldots, \mathrm{x}_{\mathrm{n}+\mathrm{k}}\right)=\sum_{i=-k}^{k} x_{n+k}(\bmod \mathrm{r})$, and a $f_{\infty}$-invariant uniform Bernoulli measure $\mu$, the measure-theoretic entropy of the additive one-dimensional cellular automata $f_{\infty}$ with respect to $\mu$ is equal to $\mathrm{h}_{\mu}\left(f_{\infty}\right)=2 \mathrm{k} \log \mathrm{r}$, where $\mathrm{k} \geq 1, \mathrm{r}-1 \in \mathrm{S}$. We also show that the uniform Bernoulli measure is a measure of maximal entropy for additive onedimensional cellular automata $f_{\infty}$.
\end{abstract}

Keywords: Cellular Automata, Measure Entropy, Topological Entropy.

\section{Introduction}

Although the additive cellular automata theory and the entropy of this additive cellular automata have grown up somewhat independently, there are strong connections between entropy theory and cellular automata theory.

We give an introduction to additive cellular automata theory and then discuss the entropy of this additive cellular automata. In [1] it was given an algorithm for computing the topological entropy of positively expansive cellular automata. For a definition and some properties of additive onedimensional cellular automata we refer to [2] (see also [1-6]). The study of the endomorphisms and the automorphisms (i.e. continuous shift commuting maps, invertible or non-invertible) of the full shift and its subshifts was initialed by Hedlund and coworkers in [3]. 
Shereshevsky has shown in [4] that if the automaton map is bipermutative then the associated CAaction is strongly mixing and it determines in every direction a Bernoulli transformation with respect to the uniform Bernoulli measure.

In [6] Ward has used (n, $\varepsilon$ )-separated sets to calculate the topological entropy of cellular automata. The definition of additive cellular automata that we have given here differs from the definition given in [6]. Detailed information about cellular automata may be found in Wolfram's paper [7].

In order to state our result, we first recall a formulation of our problem. We can also calculate the topological entropy of additive cellular automata $f_{\infty}$. We show that there is a maximal measure for additive cellular automata.

The organization of the paper is as follows: In section 2 we establish the basic formulation of problem to state our main Lemma. In section 3 we state our main theorem and prove it. Section 4 contains some remarks. In section 5 there is a conclusion.

\section{Formulation of the Problem and Definitions}

Let $S=\{0,1,2, \ldots, r-1\}$ be a finite alphabet and $\Omega=S^{Z}$ be the space of doubly infinite sequence $\mathrm{x}=\left(x_{n}\right)_{n=-\infty}^{\infty}, \mathrm{x}_{\mathrm{n}} \in \mathrm{S}$. The shift $\sigma: \Omega \rightarrow \Omega$ defined by $(\sigma \mathrm{x})_{i}=\mathrm{x}_{i+1}$ is a homeomorphism of compact metric space $\Omega$. Assume that a function $f\left(\mathrm{x}_{-k}, \ldots, \mathrm{x}_{k}\right)$ with values in $\mathrm{S}$ is given. This function generates a cellular automata $f_{\infty}$ of $\Omega$ by the formula:

$$
f_{\infty} \mathrm{x}=\left(y_{n}\right)_{n=-\infty}^{\infty}, \mathrm{y}_{\mathrm{n}}=f\left(\mathrm{x}_{n-k}, \ldots, \mathrm{x}_{n+k}\right) .
$$

Cellular automata $f_{\infty}$ is continuous and commutes with left shift (see [3]).

Definition 1. (see [2]) $f$ is additive if and only if it can be written as

$$
f\left(\mathrm{x}_{\mathrm{n}-\mathrm{k}}, \ldots, \mathrm{x}_{\mathrm{n}+\mathrm{k}}\right)=\sum_{i=-k}^{k} \lambda_{i} x_{n+i}(\bmod \mathrm{r})
$$

where $\lambda_{i} \in \mathrm{S}$. From now on, we will say that a cellular automata is additive if the local rule on which it is based is additive.

Let us consider particular case when $f\left(\mathrm{x}_{\mathrm{n}-\mathrm{k}}, \ldots, \mathrm{x}_{\mathrm{n}+\mathrm{k}}\right)=\sum_{i=-k}^{k} x_{n+i}(\bmod \mathrm{r})$. Given integer $\mathrm{s} \leq \mathrm{t}$ and a block $\left(i_{\mathbf{s}}, \ldots, i_{t}\right) \in \mathrm{S}^{(t-s+1)}$. We define the cylinder set

$$
{ }_{s}\left[i_{s}, \ldots, i_{t}\right]_{t}=\left\{x \in \Omega: x_{j}=i_{j}, s \leq \mathrm{j} \leq t\right\} .
$$

Let $\xi(\mathrm{s}, \mathrm{t})$ denote the partition of $\Omega$ into the cylinder sets of the form ${ }_{s}\left[i_{s}, \ldots, i_{t}\right]_{t}$, where $\left(i_{\mathbf{s}}, \ldots, i_{t}\right)$ runs over $\mathrm{S}^{t-s+1}$.

Lemma. Suppose that $f\left(\mathrm{x}_{\mathrm{n}-\mathrm{k}}, \ldots, \mathrm{x}_{\mathrm{n}+\mathrm{k}}\right)=\sum_{i=-k}^{k} x_{n+i}(\bmod \mathrm{r})$ and $\xi(-\mathrm{k}, \mathrm{k})$ is a partition of $\Omega$, where $\mathrm{k} \geq 2$, then the partition $\xi(-\mathrm{k}, \mathrm{k})$ is a generator for additive cellular automata $f_{\infty}$.

Proof. Let $\xi(-\mathrm{k}, \mathrm{k})=\left\{{ }_{-k}\left[i_{-k}, \ldots, i_{k}\right]_{k}: i_{-k}, \ldots, i_{k} \in \mathrm{S}\right\}$ be a partition of $\Omega$. Evidently the partition $\xi$ consist of $\mathrm{r}^{2 \mathrm{k}+1}$ elements with same measure $\mathrm{r}^{-(2 \mathrm{k}+1)}$. If we take the local rule $f\left(\mathrm{x}_{\mathrm{n}-\mathrm{k}}, \ldots, \mathrm{x}_{\mathrm{n}+\mathrm{k}}\right)=\sum_{i=-k}^{k} x_{n+i}(\bmod \mathrm{r})$, then it is easy to describe $\left(f_{\infty}\right)^{-1}(\xi)$. Let us consider a centered cylinder set $\mathrm{C}={ }_{-k}\left[i_{-k}, \ldots, i_{k}\right]_{k}$. 
Then it can be written

$$
\left(f_{\infty}\right)^{-1}(C)=\left\{\left\{\mathrm{y} \in \Omega: \mathrm{y}_{-2 \mathrm{k}}=\mathrm{j}_{-2 \mathrm{k}}, \ldots, \mathrm{y}_{2 \mathrm{k}}=\mathrm{j}_{2 \mathrm{k}}\right\}: \mathrm{j}_{-2 \mathrm{k}}, \ldots, \mathrm{j}_{2 \mathrm{k}} \in \mathrm{S}\right\},
$$

where

$\mathrm{y}_{-2 \mathrm{k}}+\ldots+\mathrm{y}_{0}=i_{-k}(\bmod \mathrm{r})$

$\cdots$

$\cdots$

$\mathrm{y}_{-\mathrm{k}}+\ldots+\mathrm{y}_{\mathrm{k}}=i_{0}(\bmod \mathrm{r})$

$\mathrm{y}_{0}+\ldots+\mathrm{y}_{2 \mathrm{k}}=i_{k}(\bmod \mathrm{r})$.

The partition $\xi \vee\left(f_{\infty}\right)^{-1}(\xi)$ consists of as following elements;

$$
\left\{\mathrm{y} \in \Omega: \mathrm{y}_{-2 \mathrm{k}}=\mathrm{j}_{-2 \mathrm{k}}, \ldots, \mathrm{y}_{2 \mathrm{k}}=\mathrm{j}_{2 \mathrm{k}}\right\} \text {. }
$$

Similarly, it is easy to show that partition $\xi \vee\left(f_{\infty}\right)^{-1}(\xi) \vee \ldots \vee\left(f_{\infty}\right)^{-(\mathrm{n}-1)}(\xi)$ consists of as following elements;

$$
\left\{\mathrm{z} \in \Omega: \mathrm{Z}_{\text {-nk }}=i_{-n k}, \ldots, \mathrm{z}_{\mathrm{nk}}=i_{n k}\right\},
$$

where $i_{-n k}, \ldots, i_{n k} \in \mathrm{S}$. Then it is evident that $\xi$ is a generator, that is, $\underset{i=0}{\vee}\left(f_{\infty}\right)^{-i}(\xi)=\varepsilon$.

Example. Let $\mathrm{S}=\{0,1\}$ and $f\left(x_{-2}, \ldots, x_{2}\right)=\sum_{i=-2}^{2} x_{i}(\bmod 2)$. Then we can write

$$
\begin{aligned}
\left(f_{\infty}\right)^{-1}\left({ }_{-2}[01101]_{2}\right) & ={ }_{-4}[111100100]_{4} \cup_{-4}[111010101]_{4} \cup_{-4}[110110110]_{4} \\
& \cup_{-4}[101110000]_{4} \cup{ }_{-4}[011111100]_{4} \cup_{-4}[110000111]_{4} \\
& \cup_{-4}[011001101]_{4} \cup_{-4}[001101000]_{4} \cup_{-4}[000111010]_{4} \\
& \cup_{-4}[101000001]_{4} \cup_{-4}[010101110]_{4} \cup_{-4}[001011001]_{4} \\
& \cup_{-4}[010011111]_{4} \cup_{-4}[100100010]_{4} \cup_{-4}[100010011]_{4} \\
& \cup_{-4}[000001011]_{4} .
\end{aligned}
$$

It is evidently $\left(f_{\infty}\right)^{-1}\left(-_{2}[01101]_{2}\right) \cap \xi$ consists of only one cylinder set.

Now we calculate the measure of the first preimage of centered cylinder set $\mathrm{C}={ }_{-\mathrm{k}}\left[\mathrm{i}_{-\mathrm{k}}, \ldots, \mathrm{i}_{\mathrm{k}}\right]_{\mathrm{k}}$ under additive cellular automata $f_{\infty}$.

$$
\begin{aligned}
\mu\left(\left(f_{\infty}\right)^{-1}(\mathrm{C})\right) & =\mathrm{r}^{2 \mathrm{k}} \mu\left\{\mathrm{x} \in \Omega: \mathrm{x}_{-2 \mathrm{k}}=\mathrm{i}_{-2 \mathrm{k}}, \ldots, \mathrm{x}_{2 \mathrm{k}}=\mathrm{i}_{2 \mathrm{k}}, \mathrm{i}_{-2 \mathrm{k}}, \ldots, \mathrm{i}_{2 \mathrm{k}} \in \mathrm{S}\right\} \\
& =\mathrm{r}^{2 \mathrm{k}} \mathrm{r}^{-(4 \mathrm{k}+1)}=\mathrm{r}^{-(2 \mathrm{k}+1)} .
\end{aligned}
$$

Similarly, we can calculate the measure of the second preimage of centered cylinder set $\mathrm{C}={ }_{-\mathrm{k}}\left[\mathrm{i}_{-\mathrm{k}}, \ldots, \mathrm{i}_{\mathrm{k}}\right]_{\mathrm{k}}$ under additive cellular automata $f_{\infty}$. It is easy to see that the second preimage of $\mathrm{C}$ has amount $\mathrm{r}^{4 \mathrm{k}}$ as centered cylinder sets

Then we have

$$
\left\{x \in \Omega: x_{-3 k}=i_{-3 k}, \ldots, x_{3 k}=i_{3 k}, i_{-3 k}, \ldots, i_{3 k} \in S\right\} .
$$

$$
\begin{aligned}
\mu\left(\left(f_{\infty}\right)^{-2}(C)\right) & =r^{4 k} \mu\left\{x \in \Omega: x_{-3 k}=i_{-3 k}, \ldots, x_{3 k}=i_{3 k}, i_{-3 k}, \ldots, i_{3 k} \in S\right\} \\
& =r^{4 k} r^{-(6 k+1)}=r^{-(2 k+1)} .
\end{aligned}
$$

If we continue this operation, in a similar way, we can determine the measure of the (n-1)st preimages of the centered cylinder set $C={ }_{-k}\left[i_{-k}, \ldots, i_{k}\right]_{k}$. It is also easy to see that the (n-1)st preimage of the centered block $\mathrm{C}$ has amount $\mathrm{r}^{2(\mathrm{n}-1) \mathrm{k}}$ as centered cylinder sets 


$$
\left\{x \in \Omega: x_{-n k}=i_{-n k}, \ldots, x_{n k}=i_{n k}, i_{-n k}, \ldots, i_{n k} \in S\right\} .
$$

We can calculate the measure

$$
\begin{aligned}
\mu\left(\left(f_{\infty}\right)^{-(n-1)}(C)\right) & =r^{2(n-1) k} \mu\left\{x \in \Omega: x_{-n k}=i_{-n k}, \ldots, x_{n k}=i_{n k}, i_{-n k}, \ldots, i_{n k} \in S\right\} \\
& =r^{2(n-1) k} r^{-(2 n k+1)}=r^{-(2 k+1)} .
\end{aligned}
$$

It is obvious that the additive one-dimensional cellular automata $f_{\infty}$ is a uniform Bernoulli measurepreserving transformation.

\section{The Measure Entropy of the Additive One-Dimensional Cellular Automata $f_{\infty}$}

In this section we introduce the measure entropy of the additive cellular automata defined above. Now we can state the main result.

Theorem. Let $\mu$ be the uniform Bernoulli measure on $\Omega$ with $p(\mathrm{i})=\frac{1}{r}$, for each $\mathrm{i}=0,1, \ldots, \mathrm{r}-1$ and $f\left(\mathrm{x}_{\mathrm{n}-\mathrm{k}}, \ldots, \mathrm{x}_{\mathrm{n}+\mathrm{k}}\right)=\sum_{i=-k}^{k} x_{n+i}(\bmod \mathrm{r})$. Then measure-theoretic entropy of the additive one-dimensional cellular automata $f_{\infty}$ with respect to $\mu$ is equal to $2 \mathrm{klog}$ r.

Proof. Now we can calculate the measure entropy of the additive cellular automata $f_{\infty}$ by using the Kolmogorov-Sinai Theorem [5], namely,

$$
\mathrm{h}_{\mu}\left(f_{\infty}\right)=\mathrm{h}_{\mu}\left(f_{\infty}, \xi\right),
$$

and $\xi$ is a generator from Lemma. It is easy to see that if

then we have

$$
\xi=\left\{\text {-k }\left[\mathrm{i}_{-\mathrm{k}}, \ldots, \mathrm{i}_{\mathrm{k}}\right]_{\mathrm{k}}: \mathrm{i}_{-\mathrm{k}}, \ldots, \mathrm{i}_{\mathrm{k}} \in \mathrm{S}\right\}
$$

$$
\begin{aligned}
H(\xi) & =-r^{(2 k+1)} \mu\left({ }_{-k}\left[i_{-k}, \ldots, i_{k}\right]_{k}\right) \log \mu\left({ }_{-k}\left[i_{-k}, \ldots, i_{k}\right]_{k}\right) \\
& =-r^{(2 k+1)} r^{-(2 k+1)} \log r^{-(2 k+1)} \\
& =(2 k+1) \log r
\end{aligned}
$$

hence

$$
\xi \vee\left(f_{\infty}\right)^{-1}(\xi)=\left\{\left\{x \in \Omega: x_{-2 k}=i_{-2 k}, \ldots, x_{2 k}=i_{2 k}\right\}, i_{-2 k}, \ldots, i_{2 k} \in S\right\}
$$

So

$$
\begin{aligned}
\mathrm{H}\left(\xi_{\vee} \vee\left(f_{\infty}\right)^{-1}(\xi)\right) & =-\mathrm{r}^{(4 \mathrm{k}+1)} \mu\left(-2 \mathrm{k}\left[\mathrm{i}_{-2 \mathrm{k}}, \ldots, \mathrm{i}_{2 \mathrm{k}}\right]_{2 \mathrm{k}}\right) \log \mu\left(-2 \mathrm{k}\left[\mathrm{i}_{-2 \mathrm{k}}, \ldots, \mathrm{i}_{2 \mathrm{k}}\right]_{2 \mathrm{k}}\right) \\
& =-\mathrm{r}^{(4 \mathrm{k}+1)} \mathrm{r}^{-(4 \mathrm{k}+1)} \log \mathrm{r}^{-(4 \mathrm{k}+1)}=(4 \mathrm{k}+1) \log \mathrm{r} .
\end{aligned}
$$

If we continue, then we have the following results:

$$
\xi \vee\left(f_{\infty}\right)^{-1}(\xi) \vee \ldots \vee\left(f_{\infty}\right)^{-(\mathrm{n}-1)}(\xi)=\left\{\left\{\mathrm{x} \in \Omega: \mathrm{x}_{-\mathrm{nk}}=\mathrm{i}_{-\mathrm{nk}}, \ldots, \mathrm{x}_{\mathrm{nk}}=\mathrm{i}_{\mathrm{nk}}\right\}, \mathrm{i}_{-\mathrm{nk}}, \ldots, \mathrm{i}_{\mathrm{nk}} \in \mathrm{S}\right\} .
$$

Finally, it is not hard show that

$$
\begin{aligned}
\mathrm{H}\left(\xi \vee\left(f_{\infty}\right)^{-1}(\xi) \vee \ldots \vee\left(f_{\infty}\right)^{-(\mathrm{n}-1)}(\xi)\right) & =-\mathrm{r}^{2 \mathrm{nk}+1} \mu\left(-\mathrm{nk}\left[\mathrm{i}_{-\mathrm{nk}}, \ldots, \mathrm{i}_{\mathrm{nk}}\right]_{\mathrm{nk}}\right) \log \mu\left(-\mathrm{nk}\left[\mathrm{i}_{-\mathrm{nk}}, \ldots, \mathrm{i}_{\mathrm{nk}}\right]_{\mathrm{nk}}\right) \\
& =-\mathrm{r}^{2 \mathrm{nk}+1} \mathrm{r}^{-(2 \mathrm{nk}+1)} \log \mathrm{r}^{-(2 \mathrm{nk}+1)}=(2 \mathrm{nk}+1) \log \mathrm{r} .
\end{aligned}
$$

So we have

$$
\mathrm{h}_{\mu}\left(f_{\infty}\right)=\lim _{n \rightarrow \infty} \frac{1}{n} H\left(\vee_{i=0}^{n-1} f_{\infty}^{-i}(\xi)\right)=\lim _{n \rightarrow \infty} \frac{2 n k+1}{n} \log r=2 \mathrm{k} \log \mathrm{r} .
$$




\section{Measures with Maximal Entropy}

The metric on $\Omega$ is defined by $\rho(x, y)=\sum_{i=-\infty}^{\infty} 2^{-|i|} d\left(x_{i}, y_{i}\right)$, where $\mathrm{d}$ is a metric on $\mathrm{S}$ and $\Omega$ is a compact metric space.

Definition 2. Let $f_{\infty}$ be any uniformly continuous map of a metric space $(\Omega, \rho)$. A set $\mathrm{E} \subset \Omega$ is said to be $(\mathrm{n}, \varepsilon)$-separated under $f_{\infty}$ if for every pair $\mathrm{x} \neq \mathrm{y}$ in $\mathrm{E}$ there is a

$\mathrm{m} \in\{0,1, \ldots, \mathrm{n}-1\}$ with the property that $\left.\rho\left(\left(f_{\infty}\right)^{\mathrm{m}}(\mathrm{x}), f_{\infty}\right)^{\mathrm{m}}(\mathrm{y})\right)>\varepsilon$. For each compact set $\mathrm{K} \subset \Omega$, let

$$
\mathrm{s}_{\mathrm{K}}(\mathrm{n}, \varepsilon)=\max \left\{|\mathrm{E}|: \mathrm{E} \subset \mathrm{K} \text { is }(\mathrm{n}, \varepsilon) \text {-separated under } f_{\infty}\right\} \text {, }
$$

$$
\mathrm{h}_{\mathrm{K}}\left(f_{\infty}, \varepsilon\right)=\limsup _{n \rightarrow \infty} \frac{1}{n} \log s_{K}(n, \varepsilon)
$$

and

$$
\mathrm{h}_{\mathrm{K}}\left(f_{\infty}\right)=\lim _{\varepsilon \rightarrow 0} h_{K}\left(f_{\infty}, \varepsilon\right)
$$

finally, define the topological entropy of $f_{\infty}$ to be $\mathrm{h}_{\mathrm{top}}\left(f_{\infty}\right)=\underset{K}{\sup } h_{K}\left(f_{\infty}\right)$. See $[1,6]$ for the topological entropy of an additive cellular automata $f_{\infty}$.

Corollary ([6, Corollary $])$. An additive cellular automata $f_{\infty}: \Omega \rightarrow \Omega$ with local rule

$$
f\left(\mathrm{x}_{-\mathrm{u}}, \ldots, \mathrm{x}_{0}, \ldots, \mathrm{x}_{\mathrm{k}}\right)=\left(\mathrm{a}_{-\mathrm{u}} \mathrm{x}_{-\mathrm{u}}+\ldots+\mathrm{a}_{0} \mathrm{x}_{0}+\ldots+\mathrm{a}_{\mathrm{k}} \mathrm{x}_{\mathrm{k}}\right)(\bmod \mathrm{r}),
$$

where $a_{-u}, \ldots, a_{0}, \ldots, a_{k} \in S$ and $a_{-u}, a_{k} \neq 0$, has topological entropy

$$
\mathrm{h}_{\mathrm{top}}\left(f_{\infty}\right)=\left\{\begin{array}{cc}
\mathrm{k} \log \mathrm{r} & \text { if } \mathrm{k} \geq-\mathrm{u} \geq 0 \\
(\mathrm{u}+\mathrm{k}) \log \mathrm{r} & \text { if } \mathrm{k}, \mathrm{u} \geq 0 \\
\mathrm{u} \log \mathrm{r} & \text { if }-\mathrm{u} \leq \mathrm{k} \leq 0
\end{array}\right.
$$

In this paper we assume that $\mathrm{a}_{-\mathrm{u}}=\ldots=\mathrm{a}_{0}=\ldots=\mathrm{a}_{\mathrm{k}}=1$.

Definition 3. The measure $\mu$ is maximal in the sense that the measure-theoretic entropy of $f_{\infty}$ with respect to $\mu$ coincides with the topological entropy $h_{\text {top }}\left(f_{\infty}\right)$.

Remark 1. Let $\mu$ be a uniform Bernoulli measure on $\Omega$ with $p(i)=\frac{1}{r}$, for each $i=0,1, \ldots, r-1$, and $f\left(\mathrm{x}_{\mathrm{n}-\mathrm{k}}, \ldots, \mathrm{x}_{\mathrm{n}+\mathrm{k}}\right)=\sum_{i=-k}^{k} x_{n+i}(\bmod \mathrm{r})$. Because measure entropy of additive cellular automata $f_{\infty}$ with respect to $\mu$ is equal to

$$
2 \mathrm{k} \log \mathrm{r}=\mathrm{h}_{\mu}\left(f_{\infty}\right)=\mathrm{h}_{\text {top }}\left(f_{\infty}\right),
$$

the additive cellular automata has a maximal measure.

Remark 2. There are $(r)^{r^{2 k+1}}$ different functions $\mathrm{f}:\left(\mathrm{x}_{\mathrm{n}-\mathrm{k}}, \ldots, \mathrm{x}_{\mathrm{n}+\mathrm{k}}\right) \rightarrow \mathrm{S}$. The function

$$
f\left(\mathrm{x}_{\mathrm{n}-\mathrm{k}}, \ldots, \mathrm{x}_{\mathrm{n}+\mathrm{k}}\right)=\sum_{i=-k}^{k} x_{n+i}(\bmod \mathrm{r})
$$

defines the cellular automata $f_{\infty}$ with maximal entropy among such functions.

\section{Conclusion}

This paper contains the following results: 
- We have found a generating partition for the additive one-dimensional cellular automata $f_{\infty}$ (Lemma).

- We have calculated the measure-theoretic entropy of the additive one-dimensional cellular automata $f_{\infty}$ (Theorem).

- We have compared the measure-theoretic entropy and the topological entropy of the additive one-dimensional cellular automata. We have seen that the uniform Bernoulli measure is a measure of maximal entropy for additive one-dimensional cellular automata.

In view of Remark 1 , an interesting open question is whether there exists a measure of maximal entropy for D-dimensional ( $\mathrm{D} \geq 2$ ) additive cellular automata over the $\operatorname{ring} \mathbf{Z}_{\mathrm{r}}(\mathrm{r} \geq 2)$.

\section{Acknowledgements}

The author acknowledges the support provided by TUBITAK, thanks Professor Nasir Ganikhodjaev for his encouragement and helpful discussion during the preparation of the manuscript. In addition, he is grateful to the referees for their many helpful comments.

\section{References}

1. M. D'amico, G. Manzini, L. Margara. On computing the entropy of cellular automata. Theor. Comput. Sci. 2003, 290, 1629-1646.

2. P. Favati, G. Lotti, L. Margara. Additive one-dimensional cellular automata are chaotic according to Devaney's definition of chaos. Theor. Comput. Sci. 1997, 174, 157-170.

3. G. A. Hedlund. Endomorphisms and automorphisms of full shift dynamical system. Math. Syst. Theor. 1969, 3, 320-375.

4. M. A. Shereshevsky. Ergodic properties of certain surjective cellular automata. Mh. Math 1992, 114, 305-316.

5. P. Walters. An introduction to ergodic theory; Springer-Verlag New York, 1982, 95.

6. T. Ward. Additive Cellular Automata and Volume Growth. Entropy 2000, 2, 142-167.

7. S. Wolfram. Statistical mechanics of cellular automata. Rev. Modern Physics 1983, 55, 601644.

(C) 2003 by MDPI (http://www.mdpi.org). Reproduction for noncommercial purposes permitted. 